\title{
PENSAR EN CIUDADES HABITABLES PARA EL FUTURO
}

\author{
Horacio CAPEL ${ }^{1}$
}

RESUMEN - Algunas reuniones organizadas por Naciones Unidas desde comienzos del milenio proporcionan un panorama de las ideas dominantes sobre la ciudad, y una buena descripción de los problemas que se plantean y las soluciones que se debaten. Eso permite reflexionar sobre los problemas de la ciudad actual y las perspectivas para el futuro. Existen grandes dificultades para el acuerdo respecto a la ciudad del presente y del futuro. Debemos expresar nuestras propias ideas sobre cómo concebimos la ciudad y como queremos que sea, formular esos deseos para el futuro, y estar dispuestos a debatirlos públicamente. Pensar en la ciudad del futuro debe partir del reconocimiento de que el futuro depende de nosotros, y que debemos presentar alternativas en relación con nuestros deseos sobre la ciudad.

Palabras clave: Problemas de la ciudad; futuro urbano; pensar el futuro.

RESUMO - Pensar em cidades habitáveis para o futuro. Algumas das reuniões organizadas pelas Nações Unidas desde os inícios do milénio proporcionam um panorama das ideias sobre a cidade, uma boa descrição dos problemas que se colocam e as soluções que se debatem. Tal permite reflectir sobre os problemas da cidade actual e as perspectivas para o futuro. Existem grandes dificuldades para um acordo a respeito da cidade do presente e do futuro. Devemos expressar as nossas ideias sobre como concebemos a cidade e como queremos que seja, formular esses desejos para o futuro, e estar dispostos a debatê-los publicamente. Pensar a cidade do futuro deve partir do reconhecimento de que o futuro depende de nós e devemos apresentar alternativas em relação aos nossos desejos sobre a cidade.

Palavras-chave: Problemas da cidade; futuro urbano; pensar no futuro.

ABSTRACT - Thoughts on livable cities for the future. Diverse meetings organized by the United Nations since the beginning of the millennium provide an overview of the dominant ideas about the city, a good description of the problems encountered and solutions discussed. This allows us to reflect on the problems of the modern city and on prospects for the future. We face great difficulties in relation to the city's present and future. We must express our own ideas on how we conceive the city and how we want it to be, to 
formulate these desires for the future, and to be willing to discuss them publicly. Thinking about the future of the city must start with the recognition that the future depends on us, and that we should present alternatives in relation to our wishes for the city.

Keywords: Problems of the city; urban future; thinking about the future.

RESUME - Penser Des Villes futures Qui Soient habitables. Diverses réunions, organisées par les Nations Unies depuis le début du présent millénaire, fournissent un panorama des idées dominantes concernant les villes et indiquent les problèmes posés et les solutions possibles. On peut ainsi réfléchir sur les problèmes de la ville actuelle et sur les perspectives futures, bien que l'accord soit fort difficile sur ces aspects. Nous devons donc exprimer nos propres idées sur ce que la ville est pour nous et sur ce que nous voudrions qu'elle soit, et formuler nos souhaits concernant l'avenir, en acceptant d'en débattre publiquement. Penser les villes futures implique d'accepter que ce futur dépende de nous-mêmes et que nous ayons donc le devoir de présenter les alternatives qui nous semblent souhaitables.

Mots clés: Problèmes urbains; futur des villes; penser le futur.

\section{PENSAR EN CIUDADES HABITABLES PARA EL FUTURO}

Hablar del futuro de las ciudades es un reto difícil, debido a la carácter imprevisible de muchos acontecimientos. Podemos examinar lo que se dice sobre la situación actual de las ciudades del mundo, y los cambios que se perciben; como vienen haciendo desde hace años diversos organismos de las Naciones Unidas. También hemos de ser conscientes de la diversidad de concepciones sobre lo que debe ser la ciudad, y de la dificultad de acuerdos. A pesar de ello, debemos esforzarnos por presentar explícitamente las alternativas que existen y nuestras propias concepciones. Hay muchos futuros posibles, pero unos son preferibles a otros, y depende de nosotros el camino que sigamos.

Las reuniones organizadas por las Naciones Unidas desde comienzos del milenio pueden facilitar un panorama de las ideas dominantes sobre la ciudad, y una buena descripción de los problemas que se plantean y las soluciones que se debaten. Aludiré primeramente a las publicaciones sobre el Estado de las Ciudades del Mundo y presentaré, después, algunas ideas básicas del último Foro Urbano Mundial. Eso nos permitirá reflexionar sobre los problemas de la ciudad actual y las perspectivas para el futuro.

Pensar en la ciudad del futuro debe partir del reconocimiento de que el futuro depende de nosotros, y que debemos presentar alternativas en relación con nuestros deseos sobre la ciudad. ${ }^{\mathrm{i}}$

\section{EL ESTADO ACTUAL DE LAS CIUDADES Y LA CIUDAD DEL SIGLO XXI}

Desde 2001 han aparecido varias publicaciones sobre State Of The World's Cities, organizadas y editadas por la ONU: la primera correspondiente a 2004-2005, se dedicó a 
Globalization and Urban Culture; la segunda, 2006-2007, versó sobre The Millennium Development Goals And Urban Sustainability: 30 Years Of Shaping The Habitat Agenda; la tercera, 2008-2009, sobre Harmonious Cities; la cuarta, 2010-11, Cities for All: Bridging the Urban Divide. La última publicación, correspondiente a 2012-2013, ha tratado de Prosperity of Cities $^{\mathrm{ii}} y$ facilita un buen panorama de las perspectivas para la ciudad del siglo XXI.

A través de dichos informes y de las publicaciones de UN-Habitat se puede tener una buena perspectiva de la evolución de las magnitudes esenciales de la urbanización. La información estadística reunida en ellas es muy valiosa, y también los estudios que los acompañan. La idea básica que aparece en el último estudio es que las ciudades tienen muchos problemas, que se estudian en detalle: desigual acceso a las oportunidades y recursos, formas antiguas y nuevas de exclusión social, marginalización y pobreza, y otros. Pero, a pesar de eso, se considera que las mismas ciudades son un factor muy positivo para superar dichos problemas, y para transformar los países, haciéndolos más desarrollados y ricos. Las valoraciones favorables son numerosas y se repiten desde la misma introducción del Secretario General de la ONU, Ban Ki-moon, y del Director de UN-Habitat, Joan Clos, antiguo alcalde de Barcelona. Se afirma explícitamente que en las ciudades se pueden encontrar "un conjunto común de condiciones que permiten a todos los residentes desarrollarse, sentirse felices y saludables; y en las que los negocios pueden florecer, las instituciones desarrollarse, y los espacios físicos se hacen más integrados y diversos". Explícitamente se consideran un remedio para las crisis globales.

Junto a ello se defiende que se necesita otro tipo de ciudad para el siglo XXI. Es preciso "reconsiderar el modelo existente de desarrollo urbano, introduciendo cambios sustanciales en la forma y función de la ciudad - un enfoque que reconfigura el espacio urbano mediante el planeamiento y diseño adecuado, creando una ciudad a escala humana en la que la diversidad, la conectividad y la integración física están todos interrelacionados y la prosperidad se comparte".

Ese nuevo espacio urbano requiere un tipo de ciudad diferente al que existe: una ciudad centrada en la gente, capaz de integrar los aspectos tangibles e intangibles de la prosperidad, haciendo desaparecer las ineficientes e insostenibles formas y funcionalidades de la ciudad de los siglos anteriores.

La visión optimista se refleja en el mismo titulo del informe que, a pesar de la crisis económica actual, centra la atención en la prosperidad de las ciudades. Se considera que éstas pueden ser un factor de prosperidad si se deciden a impulsar una nueva noción de ella, que supere el tradicional modelo de acumulación que beneficia solo a unos pocos en detrimento de otros, y si se es capaz de desarrollar otro modelo que se extiende "más allá del estrecho dominio del crecimiento económico que ha dominado las agendas mal equilibradas durante las últimas décadas". Un nuevo modelo que es "más holístico e integrado, e incluye otras dimensiones vitales tales como calidad de vida, las infraestructuras adecuadas y la sostenibilidad ambiental".

Esa ciudad del siglo XXI que se desea "trasciende la forma y funcionalidad de los modelos anteriores, equilibrando menores costes de energía con una menor huella eco- 
lógica, una forma más compacta, y una mayor heterogeneidad". Es una ciudad que trata, también, de "salvaguardarse ante nuevos peligros, y crea condiciones para una provisión más elevada de bienes públicos, junto con espacios más creativos para la imaginación y la interacción social". Y que, además, reduce la vulnerabilidad para todos, incluyendo los pobres, estimula la creación de empleo, crea armonía entre las diferentes dimensiones y proporciona las condiciones sociales, políticas y económicas de la prosperidad. Lo que tiene un corolario, formalmente expresado: "las ciudades son el remedio a las crisis globales".

El estudio clasifica a las ciudades según un índice de prosperidad, y reúne una colección de datos estadísticos sobre numerosas ciudades del mundo, las de más de 750.000 habitantes. Señala que en los países desarrollados, que ya tienen índices muy elevados de urbanización, la población urbana está casi estancada. En los países en desarrollo se reconocen patrones divergentes de crecimiento urbano. Los datos a escala global muestran a la vez la gravedad de los problemas y la disminución relativa de algunos de ellos. Por ejemplo, la población urbana del mundo que vive en las áreas de urbanización precaria (slums) de los países en desarrollo ha pasado de 650 millones en 1990, a 759 en 2000 y a 862 en 2012. Pero la proporción se ha reducido, pasando respectivamente de 46 por ciento, a 39 y a 32 respecto a la población total, en las mismas fechas. Por regiones, todas han experimentado una fuerte reducción de los porcentajes, excepto Asia Occidental donde aumenta dos puntos, y Oceanía, donde es similar en el año inicial y el final.

Hay que decir que muchas de las críticas sobre la ciudad actual y de los deseos sobre la ciudad del futuro han sido ya expresadas repetidamente por los estudios de los organismos internacionales. Por ejemplo, en el que estamos citando, se reconoce que la expansión dispersa de las ciudades en periferias sin fin es el principal factor del cambio climático, y que se necesita un transporte público eficiente. Este último estudio sobre la situación de las ciudades del mundo afirma que "en muchas ciudades el planeamiento urbano ha sido instrumentalizado por el negocio inmobiliario", y que es necesaria "una noción revigorizada de planeamiento urbano, con un nuevo sistema de valores que se basa en instituciones efectivas, leyes y regulaciones bien adaptadas, soluciones urbanas sostenibles y compromiso cívico activo en los asuntos públicos" (en el capítulo Innovating to support the transition to de City of the 21st Century). Unas declaraciones con las que no se puede estar en desacuerdo.

\section{EL PANORAMA DE LO URBANO SEGÚN EL FORO URBANO MUNDIAL}

Desde 2002 el Foro Urbano Mundial/World Urban Forum, celebrado cada dos años bajo el patrocinio de la ONU a través de UN-Habitat, ha venido debatiendo los problemas urbanos del mundo, para tratar de conseguir "un mejor futuro urbano", como se dice en el lema de la organización.

En abril de 2014 se celebró en Medellín, Colombia, el Séptimo Foro. El examen de las aportaciones realizadas y de las conclusiones a que se llegaron en las distintas mesas orga- 
nizadas, permite tener un panorama claro de cuáles son los problemas percibidos como más relevantes en el momento actual, de los diagnósticos que se hacen para abordarlos y de los acuerdos y desacuerdos existentes.

El Foro se dedicó a Urban equity in development - Cities for life, y en él participaron ministros de los países representados, parlamentarios de los diversos órganos legislativos, alcaldes de numerosas ciudades, y representantes de la sociedad civil, así como organizaciones internacionales. Cada uno de esos participantes destacaba algunas cuestiones y señalaba retos y soluciones, lo que permite tener una idea de la complejidad de los problemas existentes y de las dificultades para llegar a acuerdos que permitan solucionarlos.

\section{Diferentes niveles administrativos}

Un primer problema es la diversidad de los niveles políticos y administrativos que tienen que ver con la ciudad, y de los agentes sociales que actúan en la misma. Se percibe que los problemas urbanos son a veces de difícil abordaje y solución, por la existencia de dos o tres niveles administrativos que toman decisiones y elaboran marcos normativos que afectan a las ciudades. A los niveles nacional y local se une en muchos países un nivel intermedio, que podemos llamar regional. Aunque se oyeron muchas voces que pusieron énfasis en asegurar la colaboración entre las administraciones públicas de los diferentes niveles, y la coherencia entre las medidas adoptadas en cada uno de ellos, el mismo énfasis que se puso en estas llamadas muestra que, con frecuencia, el acuerdo no existe, o que es difícil de conseguir.

Para los ministros de los países participantes, una cuestión esencial del debate fue la necesidad de que los gobiernos centrales adopten políticas nacionales de carácter general para asegurar la equidad de las ciudades y su desarrollo.

También ellos, y mucho más otros representantes, hablaron de la urgencia de incluir a las ciudades en el proceso de toma de decisiones y en la elaboración de las medidas que les afecten. Sobre todo, aquellas que tratan de conseguir que las ciudades sean un lugar para la inclusión. Los representantes de los gobiernos nacionales admitieron que se debe animar a la participación ciudadana en el diseño de proyectos urbanos, un deseo compartido por otros muchos representantes para enfrentarse a los retos y encontrar soluciones.

Los alcaldes, por su parte, insistieron, en primer lugar, en la importancia de que ellos mismos participen en la toma de decisiones, y en la necesidad de reforzar su papel en las políticas sobre la Nueva Agenda Urbana, exigiendo que los gobiernos locales tengan mayor presencia, voz y capacidad de decisión en UN-Habitat, como "miembros activos de la elaboración de políticas en las instancias de la ONU", y en los niveles internacionales que debaten el futuro de las ciudades. Reclamaron asimismo mayor influencia en la elaboración de las políticas a nivel nacional. Destacaron que las ciudades y las áreas metropolitanas son los lugares específicos donde los problemas deben ser resueltos. Y exigieron mayores recursos controlados por la ciudad, y contar con los medios financieros para abordar el futuro. Los alcaldes destacan que lo esencial es conseguir simultáneamente 
crecimiento y equidad, y aceptaron que para ello y, en general para resolver los problemas existentes, se necesita mayor cooperación entre gobiernos locales "para pensar y desarrollar nuevos modelos de gobernanza".

Los parlamentarios, a su vez, reclamaron igualmente su papel, y el de los parlamentos de que forman parte, en las políticas sobre la ciudad, y pusieron énfasis en el nivel regional, en donde, sin duda, ellos mismos tienen más influencia. Propusieron la agrupación de regiones, para asegurar servicios organizados y equitativos para todos, y señalaron que en países con fuerte gobiernos centrales "es necesario dar poder a las regiones".

Para estos representantes, los parlamentos deben planear el futuro, pero también garantizar oportunidades equitativas en la vida diaria de las gentes. Según afirman los parlamentarios para que los habitantes de las ciudades tomen decisiones sabias y prudentes, es necesario educarles, facilitarles poder e informarles. Insisten en la necesidad de normas legislativas aprobadas por los parlamentos y de dar mayor fuerza y poder a las asambleas locales, que están en contacto estrecho con las realidades de las ciudades.

Por su parte los representantes de la sociedad civil hablaron de las prioridades que deben considerarse en la Nueva Agenda Urbana, y aprovecharon la ocasión para solicitar que dicha sociedad civil y las comunidades participen oficialmente del proceso.

Finalmente, los miembros de los organismos internacionales presentes consideraron que también éstos deben tener un papel esencial en las políticas urbanas. Sobre todo, con vistas a compartir conocimiento y dar asistencia técnica, para eliminar los fallos del mercado, y contribuir a una mayor autonomía fiscal y financiación para los agentes locales públicos y privados. Animaron a la descentralización de poderes y recursos y a la cooperación descentralizada (sobre todo Sur-Sur), y consideraron como muy importante el diálogo inter-regional.

El panorama que se obtiene a partir de todas esas declaraciones es la necesidad de la colaboración para resolver los problemas, pero también las dificultades que existen para ello, por los intereses contrapuestos que las diversas instancias reflejan.

\section{Problemas urbanos: la vivienda}

El Séptimo Foro Mundial agrupó debates sobre la equidad en el desarrollo urbano, el planeamiento y el diseño urbano para la cohesión social, los servicios básicos, los instrumentos innovadores de financiación para las autoridades locales, la resiliencia urbana, y la ciudad segura como ciudad justa y equitativa.

Leyendo las declaraciones y las conclusiones del Foro podemos tener una idea de cuáles son, para las personas que han intervenido en él, los problemas urbanos esenciales en estos momentos. Los más citados son la vivienda, las áreas de autoconstrucción y vivienda precaria (slums), el acceso a servicios básicos como agua y desagüe, el suelo urbanizado bien localizado, y los riesgos de desastres naturales,

El problema de la vivienda se consideró fundamental por prácticamente todos los participantes, como lo es también la necesidad de asegurar acceso a servicios decentes 
para todos los habitantes. Los representantes de los gobiernos nacionales consideraron que las medidas políticas deben prever ayudas financieras para la construcción de viviendas, y para el acceso a la misma.

Especial énfasis se puso en la urgencia de incluir los problemas planteados por las áreas informales en la ciudad, y de abordar la autoconstrucción y el chabolismo, mejorando las condiciones de vida de su población con acceso al agua, al desagüe y a la recogida de basuras. Como problema crítico en relación con el derecho a la ciudad se apuntó al acceso al suelo y a su tenencia, y la necesidad de reconocer la "producción social del hábitat". Se afirmó que el conocimiento es poder, lo que en relación con este problema significa apoyar la realización de una cartografía precisa de los slums. Y que en la agenda se debe "asegurar que las comunidades locales son propietarios de su propio desarrollo y de sus recursos". También se insistió en la necesidad de tomar medidas: "no más discusión sin implementación".

Se valora mucho el Programa de Mejora de las Áreas Informales (Participatory Slum Programme PSUP) puesto en marcha por la Unión Europea y desarrollado por UN-Habitat desde 2008, para mejorar las condiciones de vida de las areas urbanas mas pobres en el marco de los Objetivos del Milenio de la ONU. Se debe pasar desde la mejora de las viviendas precarias a todo lo que tiene que ver con la integración de las comunidades de áreas de barraquismo en la ciudad formal, lo que es esencial para conseguir la justicia y la equidad en el desarrollo urbano. También se exigió descriminalizar la pobreza y los movimientos sociales, y se consideró que para asegurar el éxito son convenientes las aproximaciones de abajo hacia arriba; lo cual requiere obtener datos de los slums, dar poder a las comunidades y asociarlas a todas las medidas que se adopten.

\section{El sector privado y la financiación}

Otro problema urbano frecuentemente mencionado es la necesidad de crear empleo en las ciudades, lo que se considera esencial para el desarrollo urbano sostenible. En relación con ello, y con las inversiones necesarias para resolver los problemas antes mencionados o para poner en marcha las agendas, se habló ampliamente de la financiación.

En un foro que reúne a todos los países del mundo, y que ha de tener en cuenta la economía dominante, es lógico que se dé por aceptado que la actividad económica incluye un sector privado, con el que es preciso contar. Todos parecen estar de acuerdo en ello, y los debates se dirigieron a cómo conseguir atraer la inversión privada a las ciudades para resolver los problemas urbanos y crear empleo. Muchos participantes se expresaron abiertamente sobre la conveniencia de dar facilidades a la actuación empresarial, señalando que un mayor nivel de confianza de éstas facilita la inversión. Incluso se afirmó que los gobiernos deben aprender a ser favorables a la actividad empresarial, "amistosos con los negocios" (business -friendly). También se repitió que en el mundo actual de condiciones cambiantes, los gobiernos y las empresas deben tener perspicacia para conseguir la escalabilidad, es decir la capacidad para cambiar de tamaño o configuración, para lo cual son básicas las medidas gubernamentales. 
Se destacó la necesidad de que las leyes fueran estables y seguras para la inversión, y de facilitar un ambiente favorable para la actividad inversora del sector privado. También se hicieron llamadas para que las empresas entendieran más de urbanismo para invertir en las ciudades. No como obra de filantropía, sino para pasar a un nuevo contrato social que permita la innovación y la colaboración en el desarrollo económico.

Algunas declaraciones destacaron que, además del sector privado y del público, debe concederse un papel importante al tercer sector que incluye, como es sabido, entidades voluntarias, no lucrativas, formalmente organizadas, y autocontroladas, como las organizaciones no gubernamentales, solidarias, filantrópicas y cívicas de tipo diverso. Generalmente, en dichas declaraciones se considera que los tres sectores deben colaborar para la solución de los problemas urbanos, en general, y de la vivienda en particular.

Los ministros de los gobiernos presentes aceptaron, de forma general, que "las políticas nacionales necesitan integrar la visión de la ciudad". Y consideraron que para ello debe regularse a escala nacional el desarrollo urbano, la calidad de vida, la actuación de las instituciones financieras, y la de los diferentes agentes urbanos que actúan en la ciudad.

Se reconoce una y otra vez que las ciudades tienen frecuentemente muchas dificultades para atraer inversiones. Para conseguirlo es preciso explorar algunos caminos innovadores, tales como: la colaboración público-privada, la "captura del valor del suelo", o las sinergias entre la organización de los préstamos. Se dice explícitamente que es preciso "mejorar los sistemas fiscales y bancarios existentes para atraer las inversiones privadas". También se estima que un mecanismo clave son los acuerdos financieros para mejorar los intercambios y la colaboración Sur-Sur, como algo complementario a la ayuda Norte-Sur. El papel del sector privado se consideró esencial en una era de crecimiento urbano y de crisis, así como para el éxito de la Nueva Agenda Urbana, en los próximos 20 años.

Se estimó también que las inversiones del Estado y del sector privado deben considerar explícitamente las áreas urbanas, y se señalan las prioridades para la intervención: la renovación de la vivienda y las estrategias de revitalización de las vecindades, lo que significa dar más poder al tercer sector para incluir a las familias pobres en los procesos que aseguran la seguridad de la tenencia del suelo.

\section{La equidad}

Conseguir un desarrollo con equidad y que sea sostenible fue uno de los objetivos básicos de la conferencia. Esto último fue ya un objetivo desde la conferencia de UN-Habitat II de 1996, y lo seguirá siendo también en el futuro para Habitat III que se celebrará en 2016.

La idea de equidad fue repetida por todos los participantes. Pero qué se entiende por ella puede dar lugar a diferencias matizadas. Los ministros hablaron concretamente de medidas sobre: la accesibilidad para disponer de suelo y de vivienda; el acceso a los servicios básicos como agua y desagüe, la gestión de basuras y residuos sólidos, y el mantenimiento y preservación del medio ambiente. También destacaron decididamente la necesidad de políticas nacionales para conseguirla. 
Para reducir la desigualdad se coincide en la importancia de hacer las inversiones adecuadas en infraestructuras sociales y físicas. Y ampliar el campo de la participación y el compromiso. En concreto, respecto a las mujeres, es esencial considerarlas no como beneficiarias sino como participantes, lo que significa comprometerlas en la planificación y puesta en marcha de las medidas. También se habló de la necesidad de tener en cuenta a los jóvenes y a los minusválidos.

Hubo coincidencias en la necesidad de poner el foco más allá de lo urbano, y considerar la equidad como una distribución social de oportunidades, con el objetivo de que los beneficios alcancen todos. Repetidas veces se habló de la conveniencia de mejorar el conocimiento, las destrezas y las capacidades sobre la ciudad y su gestión, a todas las escalas, y de compartir todo ello. Se aludió asimismo a la creación de ciudades "inteligentes y que tengan en cuenta los valores", y a la utilización y comprensión de la tecnología por parte de todos y para todos.

Las políticas urbanas exigen cogestión y corresponsabilidad. Se repitió que es preciso mejorar las instituciones locales para aumentar su capacidad, y la urgencia de promover la transparencia y la confianza de los ciudadanos En todo caso, se percibe la falta de capacidad técnica y financiera de muchos gobiernos locales, y la necesidad de diseñar programas para mejorarlas. Es importante eliminar las distancias en el conocimiento a escala local, lo que requiere, además, promover la inversión financiera y técnica. La colaboración de todos los agentes o interesados en la ciudad se consideró una y otra vez necesaria, pero se reconoce que es muy difícil, sin duda por los objetivos distintos que tienen.

Todas las organizaciones internacionales presentes aceptaron que la urbanización tiene un papel complementario al mandato de todas ellas. Manifestaron que desean participar no sólo en las sesiones de las conferencias sino también en su preparación. Algunos señalaron que es preciso contar con las grandes ciudades, pero también con las intermedias o emergentes, que son claves pues crecen más rápidamente y están propensas a mayores desigualdades.

Los ministros destacaron que "los gobiernos centrales tienen un papel importante en el desarrollo de políticas hacia la mayor equidad", tan importante como el de los gobiernos locales; aunque reconocen que hay muchos actores que deben comprometerse con las acciones para conseguir los objetivos de equidad urbana. Lo cual plantea - dicen - "la cuestión de los recursos disponibles para cada nivel del gobierno, capacidad técnica e institucional para las entidades públicas y privadas, las ONG y la academia”. También consideran que en todo caso, sigue siendo esencial la capacitación de los elegidos y de los funcionarios del sector público.

\section{Desarrollo sostenible y planeamiento}

En relación con el desarrollo sostenible se citan los riesgos naturales, aunque la atención máxima se pone en el cambio climático, una situación nueva que exige una aproximación integrada más que sectorial. 
Entre las prioridades que se señalan para la Agenda Post-2015 está el énfasis en la sostenibilidad a escala regional "para reforzar la resiliencia frente al cambio climático", es decir, la capacidad de hacer frente a la adversidad que se ha producido; pero no se dice nada de cómo luchar contra este cambio climático y los factores que lo provocan.

No dejan de encontrarse algunas declaraciones sorprendentes; como ésta: el cambio climático "es una oportunidad para las ciudades para mejorar las infraestructuras disponibles de forma que reduzca las necesidades de financiación a largo plazo".

La nueva situación existente y la crisis económica, que es la crisis de las políticas neoliberales, han conducido a la aceptación de que el planeamiento es necesario, frente a las anteriores ideas dominantes que lo cuestionaban.

Un hecho destacado del World Urban Forum 7 es que los ministros reconocen que la crisis financiera afecta a las políticas de la vivienda. Pero también que es una "crisis del planeamiento". Añaden que "el planeamiento urbano es un instrumento de desarrollo económico".

Los alcaldes también consideran que para una urbanización sostenible son necesarios el diseño urbano y la planificación del espacio público; en la planificación urbana debe incluirse el desarrollo económico con equidad. Los alcaldes insisten en la importancia de poner énfasis en la lucha contra la corrupción como elemento de apertura y transparencia. Unos y otros declaran que hay que hacer más con menos, debido a la crisis: conseguir sinergias y tecnologías adaptables; por ejemplo: para limpiar el agua, la obtención de energía a partir de los residuos, la forma como se despliega y organiza la política para prevenir el delito. A veces se unen a esas declaraciones deseos retóricos que no tienen una concreción, aunque muestran la confianza en la realidad urbana; como esta afirmación de los ministros: "la ciudad debe ser considerada como una solución más que como un problema". Lo que es una constatación del dinamismo de las ciudades y de los beneficios económicos derivados de su crecimiento.

\section{LA RESPONSABILIDAD DEL CAPITALISMO}

En los diagnósticos realizados por las entidades internacionales hay, como hemos visto, declaraciones de confianza en las ciudades, consideradas como un factor positivo para superar los problemas, para mejorar las condiciones de vida de la población, e incluso como remedio para la crisis. Pero al mismo tiempo, se reconocen muchos aspectos negativos y numerosos problemas que existen en ellas; se habla de que hay un acceso desigual a las oportunidades y a los recursos, se mantienen formas antiguas y nuevas de exclusión social, está muy extendida la marginalización y pobreza, se denuncia la falta de vivienda, la existencia de áreas de autoconstrucción y vivienda precaria, la falta de cohesión social.

Sin duda es mucho lo que hay de acierto en estas descripciones y diagnósticos de los organismos de las Naciones Unidas, y en la conclusión de que es urgente tomar medidas para resolver dichos problemas. Incluso se alude alguna vez a la necesidad de reconsiderar el modelo económico y social existente, aunque sin mayores especificaciones. Son, en 
concreto, mínimas las alusiones a las estructuras económicas, o a las políticas neoliberales que se han venido aplicando en muchos países en las últimas décadas, y que están en la base de numerosos problemas de los países y de las ciudades.

Las referencias a la escasez de capitales para invertir en la ciudades y en muchas regiones y países están reiteradamente presentes en los diagnósticos. Y son sin duda acertadas, sobre todo en el caso de muchas grandes ciudades de países menos desarrollados; también en la mayor parte de las medias y pequeñas, menos atractivas para el capital.

Se percibe una obsesión y una lucha de las ciudades para atraer nuevas inversiones. Es grande la competencia para atraer capital en un mundo globalizado, en donde todas las ciudades compiten con todas. Pero en ocasiones, no es la falta de capitales sino su abundancia y la forma como se invierte en las ciudades, lo que está en la base de muchos problemas.

En los diagnósticos realizados por las entidades internacionales hay una expresión que está ausente: el capitalismo; éste, sin embargo, aparece como responsable de muchos problemas del mundo contemporáneo en los estudios que se realizan por científicos sociales y por los movimientos ciudadanos.

La inversión en la ciudad se ha convertido en un aspecto muy atractivo para el capital que busca nuevos negocios. El capital financiero penetra en todo, y busca nuevos sectores o lugares para el beneficio. La producción de la ciudad puede incrementar dichos beneficios a través de procesos de cambio de uso del suelo, mejorando la diferencia potencial de beneficio económico del capital.

Desde hace décadas muchas ciudades han realizado esfuerzos para facilitar la inversión de capitales, con medidas diversas, entre las cuales se encuentran: la privatización de servicios públicos, la planificación estratégica, los megaproyectos y la arquitectura espectáculo. En general, se ha tratado de lograr la revitalización de los centros urbanos, atrayendo nuevas actividades a ellos (a veces en detrimento de las tradicionales, como los comercios de vecindad o los talleres), y haciendo esas áreas más atractivas para las clases medias y altas que se desplazan allí.

En relación con todo ello, parte de la ciudad se privatiza o se vende para el negocio privado. Hay también numerosos casos de venta de edificios o de parte del suelo urbano por las administraciones municipales, regionales o estatales, con la excusa de las dificultades financieras producidas por la crisis. Ello representa siempre más oportunidades para el negocio privado y puede suponer la expulsión de la población y las actividades tradicionales, la puesta en marcha de políticas urbanas que se dirigen a atraer gentrificadores y turistas a expensas de la población tradicional. El paisaje, la historia, las características locales, la cultura, se incorporan en la mercadotecnia urbana y se promocionan al exterior para el beneficio privado.

La desvalorización o estigmatización del territorio puede servir para preparar la expulsión y la inversión, ya que mejora el beneficio diferencial del capital. La regeneración urbana se ha convertido en una estrategia central en la actuación del capital financiero. Por ejemplo, activando lo que se ha llamado gentrificación por los equipamientos, por las inversiones en parques y jardines, o por las mejoras ecológicas. 
Las grandes operaciones que a veces interesan al capital privado, muchas veces son apoyadas por los gobiernos municipales, regionales o estatales. Porque se piensa en los beneficios que van a producir. En ese caso, los inversores se aprovechan de dichas mejoras para invertir en esos espacios y obtener mayores beneficios.

Que los grupos de menores rentas no tengan acceso a los servicios básicos se debe, frecuentemente, al hecho de que no pueden pagar el precio de esos servicios, a no ser que se construyan por la administración pública. Pero las políticas de privatización de dichos servicios han sido nefastas, ya que, debido a la escasa solvencia económica de dichos grupos, nuevamente ha sido el Estado quien ha tenido necesidad de asegurar los servicios para ellos.

\section{PENSAR LA CIUDAD DEL FUTURO}

Hoy se tienen datos suficientes sobre los cambios que se han producido en el mundo, así como de las graves amenazas que existen. Lo que nos obliga a tomar medidas urgentes y pensar en el futuro. Hay quienes estiman que esto es imposible, porque entramos en el dominio de lo imprevisible. Pero el desarrollo durante el siglo XX de los "estudios sobre el futuro", muestra que es posible hacerlos y que pueden ser un camino fructífero. Desde los informes del Club de Roma en los años 1960, existe ya un campo de reflexión sobre ello entre los científicos sociales. Aunque muchas cosas son hoy muy distintas, el conocimiento de algunas situaciones del pasado, y de sus complejidades, nos puede ayudar a entender el presente y a mirar al futuro

Los "estudios del futuro" están hoy ampliamente realizados por grupos de investigación, asociaciones, instituciones gubernamentales y académicas dedicadas a ello en numerosos países. Las técnicas que se utilizan son variadas: desde extrapolación de tendencias, y simulaciones cuantitativas o narrativas, a prospecciones, proyecciones, examen de escenarios y otras, aplicadas al futuro global del mundo, a partes del mismo, o al estudio de futuros sectoriales concretos, desde la población a la economía o la tecnología, pasando por la salud, la organización social o el desarrollo urbano. Se puede tratar de imaginar cómo será el mundo del futuro haciendo simulaciones, o imaginar escenarios posibles que permitan reflexionar sobre las tendencias existentes y sus consecuencias.

El futuro está siendo pensado por muchos en estos momentos, y hay numerosos políticos y técnicos que toman decisiones para configurarlo de una determinada manera. Por eso es importante que piensen en él también los científicos sociales y los ciudadanos en general.

Aunque las variables que es preciso integrar para pensar en el futuro son muy numerosas, es posible empezar a hacer simulaciones sencillas y darles cada vez mayor complejidad. Los científicos sociales, y en especial los historiadores y los geógrafos, podrían estar especialmente preparados por el hábito que tienen para enfrentarse a situaciones complejas y con múltiples interacciones en todos los sentidos, así como para identificar las continuidades y lo que es nuevo. En todo caso, se trata de hacer juicios provisionales que 
faciliten la reflexión y el debate, y ayuden a tomar decisiones a los políticos, a los técnicos y a los ciudadanos.

Muchas ciudades tienen siglos e incluso milenios de antigüedad, en ocasiones más que los Estados. Por tanto se pueden estudiar procesos de muy larga duración. El estudio del pasado, y de diferentes situaciones complejas del mismo y del presente, puede ayudar a entender lo que sucede hoy y lo que puede ocurrir. Se pueden hacer simulaciones de lo que sucedería si se producen algunos cambios y se mantienen otros factores. Por ejemplo, qué ocurrirá si no se hace nada para frenar las migraciones irregulares, o qué si se toman medidas para frenarlas; o si se suprimen las fronteras. Que ocurrirá si no se hace nada para frenar el cambio climático, o qué si se toman algunas determinadas medidas.

También podemos imaginarnos como hombres del futuro que miran hacia el pasado y se preguntan porqué no tomamos ciertas medidas, ahora que podemos hacerlo, para detener procesos que están afectando gravemente al planeta Tierra o a la evolución de la Humanidad. Por ejemplo, porqué no hacemos nada urgente para detener el cambio climático o para llegar a acuerdos en conflictos que pueden destruir la Humanidad.

\section{SE HA PERDIDO EL SENTIDO DE LA MESURA}

Es evidente que el mundo está al borde del desastre. Nos podemos limitar a destacar tres rasgos que lo indican: la huella ecológica insostenible, el calentamiento que da lugar al cambio climático, las desigualdades sociales y los conflictos que esto provoca y puede provocar.

Seguramente el hecho más característico del mundo actual, y la raíz de muchos males es que se ha perdido el sentido de la autolimitación, de la contención, de la mesura.

Desde los años 1960, cuando empezaron a percibirse las consecuencias negativas de la pretensión de crecimiento ilimitado, existen debates y aportaciones de ideas que muestran la locura en que hemos ido a parar con las pretensiones de crecimiento ilimitado. Las propuestas de decrecimiento, que se han difundido en los últimos años son una consecuencia de dichos debates.

Esta desmesura puede tener raíces intelectuales en la cultura occidental. Pero el responsable más destacado de lo que ocurre hoy, de esta falta de mesura es el sistema económico capitalista. La búsqueda del máximo beneficio conduce a la producción masiva y, como consecuencia, a la creación de la sociedad de consumo.

La lógica económica de la producción, la competencia despiadada entre empresas, la búsqueda obsesiva de lucros, unida al aumento de la capacidad de compra de amplios grupos sociales, ha permitido la aparición de la sociedad de consumo. Se consume mucho más que en el pasado y, con frecuencia, excesivamente. Los intereses de la producción capitalista hacen que muchas mercancías se programen para que no duren, para que sean efímeras, como ya anunciaron Marx y Engels en el Manifiesto comunista, y recordó Marshall Berman en su libro Todo lo sólido se desvanece en el aire. 
La variedad de productos es excesiva; debería haber menos y de calidad mayor. Hay quien defiende que más es mejor que menos, que la diversidad de opciones, la abundancia de objetos para elegir aumenta la libertad y la capacidad de realización de los humanos, la posibilidad de experimentar y de ser feliz. Pero las consecuencias están siendo muy negativas, para el ambiente y para los seres humanos. La diversidad de objetos y la posibilidad de consumir artículos muy diversos conduce al despilfarro.

Hay innovaciones de consumo que se han difundido ampliamente y que tienen consecuencias muy negativas. Por ejemplo, muchas de las que se relacionan con el uso de plásticos para envases de productos, y que son desechables, como botellas, envoltorios y bolsas. Cada año miles de millones de bolsas de plástico acaban convertidas en basura y contaminando la naturaleza. El problema es realmente grave y el Parlamento Europeo aprobó a fines de abril de 2015 una normativa que pretende reducir el consumo de bolsas de plástico de un solo uso, imponiendo fuertes impuestos: en 2010 cada europeo utilizó 198 bolsas de plástico, y se pretende reducirlas a 90 a fines de 2019 y a 40 en 2025.

Las políticas económicas ante la crisis actual tratan, a veces, de estimular el consumo; frente a ello, quizás habría que moderarlo, ser más frugal. Es esencial la autolimitación, la contención, la moderación, la reducción del consumo. Se ha de luchar contra la obsolescencia programada, y conseguir limitar el consumo de los ciudadanos que más tienen. Solo con ello se podrá reducir la huella ecológica. Frente al crecimiento económico, seguramente es preciso promover el crecimiento de suma cero, que equilibre las pérdidas y ganancias a escala mundial.

\section{LAS DIFICULTADES PARA EL ACUERDO RESPECTO A LA CIUDAD DEL PRESENTE Y DEL FUTURO}

Pero, además de todo ello, debemos expresar deseos para la ciudad del futuro. A partir de la explicitación previa de nuestras propias ideas sobre cómo concebimos la ciudad $\mathrm{y}$ como queremos que sea. Hemos de atrevernos a formular esos deseos para el futuro, $y$ estar dispuestos a debatirlos públicamente. Sin duda, esa formulación explícita y pública contribuye, aunque sea mínimamente, a construir el futuro de la ciudad, y de la sociedad.

La ciudad actual recibe diversos calificativos, unos negativos y otros positivos, según las características esenciales que se destacan en ella. Entre los primeros: ciudad fragmentada, desigual, segregada, conflictiva, carcelaria, amnésica... Entre las calificaciones positivas: innovadora, culta, saludable... También se han inventado calificativos metafóricos que expresan los rasgos de algunas ciudades especialmente significativas, como ciudad creativa, o ciudad inteligente. En todo caso, se trata de calificativos que expresan algunos rasgos, en modelos descriptivos generales o válidos para determinadas áreas. Algunos son considerados iconos para la ciudad deseable.

Hemos de ser conscientes de que no es fácil llegar a consensos sobre el futuro que hemos de construir, porque las actitudes sobre el presente o el futuro dependen de posiciones ideológicas, y debemos ponernos previamente de acuerdo sobre cuáles son los 
futuros deseables y como se alcanzan. En lo que se refiere al futuro de la ciudad, es difícil imaginar entendimiento entre los defensores de las ideas neoliberales, y los que se oponen a ellas; entre los que magnifican lo privado y el mercado y quienes lo cuestionan y defienden la necesaria regulación por la administración pública; entre quienes consideran que la sociedad ha de estar gobernada centralizadamente o los que defienden la descentralización y el aumento de la participación ciudadana; entre quienes postulan la propiedad privada y los que estiman que debe ser colectiva o pública; entre los que valoran la importancia de aumentar el consumo individual y los que prefieren una vida más modesta y frugal que proteja el planeta. También hay diferencias importantes respecto al papel de la mujer en la sociedad, sobre la confianza en la tecnología, sobre el significado que debe atribuirse a la religión, sobre el crecimiento sostenible, sobre la desigualdad y segregación social, sobre el orden público en la ciudad las políticas represivas que disminuyen los derechos y libertades de los ciudadanos, y otras numerosas cuestiones.

Hay quienes valoran el consenso o la convivencia en paz, aunque hay sociedades o grupos políticos que se oponen a ello, como vemos por las noticias diarias de la prensa. También existen valoraciones diferentes de la democracia, y de la existencia de mecanismos formales para ejercerla. Las diferencias ideológicas son profundas; pero también las que existen en los gustos y en las valoraciones personales, que se reflejan en las actitudes diferentes que existen ante las mismas edificaciones y los diseños de planos urbanos.

Un primer acuerdo que es ya difícil de alcanzar es el referente a la necesidad de limitar el papel del mercado y de lo privado y de dar la preeminencia a la administración pública. La ordenación de la ciudad necesita de abundantes recursos públicos, lo que a su vez exige una fiscalidad adecuada. Se habla poco de ello en los foros y publicaciones de las Naciones Unidas, que antes se han citado.

La normativa urbanística tradicionalmente declaraba los espacios que podían urbanizarse, y preveía la construcción de las infraestructuras básicas. Las políticas neoliberales, en muchos países, han considerado que todo el territorio es urbanizable a no ser que se declare protegido por alguna razón. Ello ha llevado a una extensión abusiva de la urbanización, por iniciativa privada, sin que se construyeran simultáneamente las infraestructuras y equipamientos necesarios. Es evidente que esa forma de considerar todo el territorio urbanizable debe ser rechazada, y que se impone que la urbanización se realice a través de un planeamiento de control público.

Ya hemos visto que uno de los problemas reconocidos en las ciudades es la falta de viviendas. La dotación de alojamientos para todos exige la construcción de vivienda social por las administraciones públicas. Para lo cual se requiere financiación y una política fiscal bien diseñada. Pero no es suficiente. Se han de elaborar también criterios justos y aceptados para el acceso a dichas viviendas y la asignación de las mismas a las familias y los individuos. También hay que debatir si deben elaborarse políticas para facilitar la mezcla social, las ayudas a las familias, las políticas demográficas, la modernización cultural.

Se ha de preferir la ciudad compacta frente a la dispersa, que es mucho más costosa. Pero es preciso hacer un amplio debate sobre una y otra, así como sobre el consumo ener- 
gético y el coste de la creación de servicios públicos como agua, desagüe, redes eléctricas, transporte público. Asimismo, sobre la necesidad que tienen las ciudades de disponer de espacio público para todos los ciudadanos, donde nadie pueda ser rechazado o excluido, y que facilite la convivencia y la interacción. La ciudad, por otra parte, no puede aceptar la existencia de condominios, barrios o pueblos cerrados; todo el espacio urbano ha de ser abierto para todos.

El transporte público y colectivo es esencial para el buen funcionamiento de la ciudad, por lo que se debería limitar el transporte individual. También la sanidad pública es esencial, y la escuela pública de calidad para todos es la única garantía de igualdad y de convivencia en el futuro.

La contaminación, y de manera esencial la producida por las ciudades, es responsable del cambio climático. Se está también reduciendo la biodiversidad en el planeta, y es preciso limitar la huella ecológica. Se necesita urgentemente poner en marcha políticas de gestión y conservación de recursos a escala mundial. En las áreas urbanas hay que establecer sistemas de planificación que permitan mantener los paisajes más ecológicamente sostenibles. Es preciso tener en cuenta, además, que muchas ciudades están localizadas en áreas de rica diversidad y su forma de crecimiento tiene efectos negativos sobre ésta.

Las actuaciones del capital en los centros de las ciudades, y otras transformaciones urbanas son presentadas por los medios como algo positivo. Esos mismos medios acostumbran a presentar las resistencias que se producen de forma negativa. Sin embargo esos movimientos locales contra las políticas neoliberales y sus efectos expresan formas de resistencia muy importantes para el futuro (movimientos okupa, contra los desahucios y formas diversas de desposesión de la vivienda, las luchas contra el aumento de las desigualdades sociales provocadas por la crisis...). Ponen en cuestión el modelo dominante, y pueden llevar a otras formas de producir el espacio urbano.

Se necesita un acuerdo sobre una ética social consensuada, ya que la moral ha de quedar para las conciencias personales. Para resolver muchos problemas que existen, desde la corrupción a la actitud ante las minorías, se necesitan acuerdos y medidas políticas, más allá de la ética personal. Las religiones, que pudieron tener aspectos positivos en el pasado, tienen hoy a veces efectos negativos, como consecuencia, especialmente, de la actuación de las burocracias religiosas y por los fundamentalismos sectarios y dogmáticos. Tal vez sea llegado el momento de imponer en las ciudades equipamientos conjuntos para todas las religiones que adoran a un Dios que, si existe, ha de ser uno, aunque se presente por razones culturales bajo advocaciones distintas.

\section{Las cuestiones jurídicas y el derecho para la ciudad}

Los problemas de las ciudades son muchos. Pero se pueden intentar resolver con la legislación.

Desde 1968, con la publicación de la obra de Henri Lefébvre, la reivindicación del derecho a la ciudad se ha convertido en un objetivo de movimientos sociales y de congresos internacionales, que han ido llenando de contenido el mismo; se ha convertido en 
una aspiración para que toda la población del mundo tenga los niveles de vida y libertad que se encuentran en las ciudades, así como en un derecho de ciudadanía. Tal como se ha ido definiendo, por ejemplo en el congreso de Quito en 2004, el derecho a la ciudad incluye toda una serie de aspiraciones que se extienden a la salud, la educación, el empleo, el salario, los derechos civiles y políticos de los habitantes, la gestión democrática de la ciudad, la protección de grupos y personas vulnerables, el derecho al espacio público y otros.

Los problemas de las ciudades se pueden resolver con el derecho y con la aprobación de leyes. En un Estado democrático las leyes dependen de nosotros: pueden ser muy avanzadas y progresistas, e incluso revolucionarias, cuando las elabore y apruebe una mayoría política de este carácter elegida por los ciudadanos.

La normativa legal y el planeamiento urbano pueden contribuir a un futuro mejor. En una sociedad compleja como la actual, hemos de pensar sobre la forma de elaborar dicha normativa y los planes, cómo se toman las decisiones y se arbitra entre los intereses contrapuestos que pueden tratar de influir en el diseño de dichas normas, el papel atribuido a los diferentes agentes que son esenciales en la construcción de la ciudad y a las diversas instancias de la administración pública, desde la escala local a la estatal y supranacional, y de los diversos organismos que la componen, no siempre con objetivos similares. Hemos de profundizar la democracia con la participación ciudadana, siendo conscientes de sus dificultades y de las posibilidades de manipulación.

La inviolabilidad de la propiedad es hoy generalmente un dogma, pero esto puede cambiar. Para empezar, se puede plantear la cuestión de hasta cuándo se puede transmitir la propiedad de los bienes. En el caso de la propiedad de una vivienda familiar, se puede aceptar fácilmente que no más allá de los nietos o bisnietos, aspecto en el que podría encontrarse fácilmente un amplio acuerdo. Ello permitiría plantear a continuación la transmisión de la propiedad individual de todo tipo.

También es preciso llegar a acuerdos sobre el papel atribuido a lo que existe, al espacio ya construido, al patrimonio edificado del pasado. Y también de los espacios no edificados todavía, por los que se expansionará la ciudad, la atención a la larga historia del territorio, con características específicas, servidumbres, limitaciones que deben respetarse y mantenerse. El despilfarro se manifiesta también en la producción de la ciudad, de los edificios, de los entornos urbanos y de los equipamientos que la constituyen. También en todo ello se ha de pedir contención y mesura.

Las estructuras de gobierno de las ciudades, el papel del municipalismo, y su relación con las entidades supramunicipales (por ejemplo, metropolitanas), con las regionales, las estatales y las supraestatales; sobre cómo se efectúa el concierto entre las diversas instancias citadas para la gestión del suelo y la construcción de la ciudad. Existe hoy una cierta tendencia a la descentralización de los países, y la atribución, o 'devolución' de competencias a las ciudades. Algún gobierno europeo va dando pasos en esa dirección, con la transferencia a las ciudades de competencias en materia de urbanismo y vivienda, transporte, sanidad, para ayudar a resolver los problemas y reequilibrar el crecimiento económico. 
Existe hoy una movilidad geográfica muy amplia de la población mundial.

Solo la cifra de turistas que se mueve a escala internacional impresiona, pues supera ya los 1.300 millones de personas, una de cada siete del mundo. Lo que asombra mucho más porque en 1950 la cifra de turistas internacionales era de 26 millones.

La movilidad como migrantes es todavía mayor. Frente a ella, se refuerzan las fronteras y se levantan muros, y se producen continuamente tragedias en el Mar Mediterráneo, en la frontera o en el trayecto a Estados Unidos y en otros lugares. Recientemente han sido conocidos los problemas de los refugiados procedentes de Myanmar (la antigua Birmania) y de Bangaladesh hacia países del Sureste de Asia e Indonesia, cuyas autoridades los devuelven al mar, a pesar de que huyen de la pobreza y de los ataques a las minorías por razones religiosas. Son emigrantes desesperados o a los que se deben ofrecer ayuda humanitaria, aunque otros alegan que ello aumentará el volumen del éxodo; "no podemos cuidarles ¿dónde los ponemos?" ha preguntado un político tailandés.

Desde hace tiempo existe una reclamación de la ciudadanía universal, que expresaron argumentadamente ya en el siglo XVIII filósofos como Locke y Kant. Recientemente filósofos, políticos y científicos sociales han aludido a esos y otros precedentes y han defendido la ciudadanía universal como un imperativo ético y de justicia; entre ellos José Manuel Bermudo, en varios de sus libros recientes.

Esa reclamación es un ideal de contenido revolucionario. Pero ¿cómo se consigue? ¿cuánto cuesta? ¿quién la paga? ¿cómo se gestiona? Podemos imaginar decenas e incluso millones de migrantes reclamando otras nacionalidades. Y debemos pensar en los problemas que eso plantea.

Hemos de valorar las prácticas sociales urbanas, las formas de solidaridad y de colaboración. Se ha de tener confianza en la fuerza de los ideales, y atrevernos a expresarlos para el futuro. Tal vez sea útil releer las utopías, para elaborar otros ideales. A ello se dedicará el XIV Coloquio Internacional de Geocrítica.

El pasado es inconmovible, como señaló un poeta griego, y recordó Aristóteles en la Etica Nicomáquea (1139b 10): "De una cosa solo Dios está privado/de hacer que no se haya realizado lo que ya está hecho". Pero en cambio podemos influir en el futuro, modestamente con la acción individual y, de forma más intensa, con la acción colectiva. También sabemos que hay muchas vías posibles de desarrollo y muchos futuros posibles, y que algunos son más probables que otros. Y que podemos pensar en esos futuros posibles, y adoptar decisiones y comportamientos para que algunos de ellos sean más posibles, para tratar de evitar los futuros indeseables. Es posible ayudar a tomar medidas para facilitar ciertas evoluciones y evitar otras, pensar en el futuro, y empezar a prepararlo desde hoy y desde aquí. Hemos de proponer alternativas. Hay futuros que son preferibles a otros; $y$ depende en buena parte de nuestras acciones caminar hacia un futuro concreto entre los muchos que existen. 


\section{BIBLIOGRAFÍA}

Aristóteles (1985). Ética Nicomáquea. Ética Eudemia. Edición de Emilio Lledó. Traducción y notas Por Julio Pelli. Madrid: Gredos.

Bauman, Z. (2001). Globalitazió. Les conseqüécies humanes. Barcelona: Edicions de la Universitat Oberta de Catalunya.

Berman, M. (1988). Todo lo solido se desvanece en el aire. La experiencia de la modernidad. Traducción de Andrea Morales Vidal Madrid: Siglo XXI Editores.

Capel, H. (1971). Las dificultades del análisis interdisciplinar del crecimiento urbano. Revista de Geografía, V, 123-136. Reproducido en el volumen Urbanismo y práctica política. Barcelona: Los Libros de la Frontera, 1974, 9-28. http://www. raco.cat/index.php/RevistaGeografia/article/ view/45853/56647

Capel, H. (1975). La definición de lo urbano. In "Homenaje al Profesor Manuel Teran”, Estudios Geográficos, 138-139, Madrid, 265-301. Reproducido en Scripta Vetera, Universidad de Barcelona, no 33. http://www.ub.es/geocrit/sv-33.htm y en http:// www.cidadeimaginaria.org/eu/Urbano.pdf

Capel, H. (2009). La historia, la ciudad y el futuro. Scripta Nova. Revista Electrónica de Geografía y Ciencias Sociales, XIII, 307. http://www.ub.es/ geocrit/sn/sn-307.htm

Capel, H. (2010). Urbanización Generalizada, derecho a la ciudad y derecho para la ciudad. Conferencia inaugural del XI Coloquio Internacional de Geocrítica. Scripta Nova. Revista Electrónica de Geografía y Ciencias Sociales, XIV, 331 (7). http:// www.ub.es/geocrit/sn/sn-331/sn-331-7.htm
Capel, H. (2014). El futuro de las ciudades. Una propuesta de manifiesto. Biblio 3W, Revista Bibliográfica de Geografía y Ciencias Sociales, IX, 551. http://www.ub.es/geocrit/b3w-551.htm

Lefebvre, H. (1969). Le droit à la ville. Paris 1968. Versión española El derecho a la ciudad. Prólogo de Mario Gaviria. Traducción de J. González-Pueyo. Barcelona: Península.

Naciones Unidas. (2014). Objetivos del Milenio. Informe de 2014. New York. http://www.un.org/es/ millenniumgoals/pdf/mdg-report-2014-spanish. pdf

Un-Habitat. (2001). The State of the World's Cities Report 2001 Un-Habitat. (2012). State of the World's Cities Prosperity of Cities: 2012/2013.

Un-Habitat. (2004). Globalization and Urban Culture 2004/2005. United Nations Human Settlements Programme.

Un-Habitat. (2006). State of the World's Cities The Millenium Development Goals and Urban Sustainability 2006/2007. 30 Years of Shapping the Habitat Agenda. United Nations Human Settlements Programme.

Un-Habitat. (2008). State of the World's Cities Harmonious Cities 2008/2009.

Un-Habitat. (2010). State of the World's Cities Cities for All: Bridging the Urban Divide: 2010/2011.

World Urban Forum. (2014). World Urban Forum VII (WUF 7), Urban equity in development. Cities for life. Colombia: Medellin. http://wuf7.unhabitat. org/

i Texto preparado para la VII Conferencia Anual do IGOT, Planear cidades habitáveis no século XXI: Saúde, Ambiente e Coesão, Universidadade de Lisboa, 21 de Maio de 2015, Sessão III, “O futuro das cidades".

ii http://www.goodreads.com/book/show/17168108-state-of-the-world-s-cities-2012-2013 